\title{
A Phase 3 study of atezolizumab as monotherapy or combined with chemotherapy vs placebo + chemotherapy in previously untreated locally advanced or metastatic urothelial carcinoma: IMvigor130
}

José Ángel Arranz Arija, ${ }^{1}$ Matthew D. Galsky, ${ }^{2}$ Maria De Santis, ${ }^{3}$ lan D. Davis, ${ }^{4}$ Aristotelis Bamias, ${ }^{5}$ Ugo De Giorgi, ${ }^{6}$ Marina Mencinger, ${ }^{7}$ Eiji Kikuchi, ${ }^{8}$ Xavier García-del-Muro, ${ }^{9}$ Mahmut Gumus, ${ }^{10}$ Mustafa Özgüroğlu, ${ }^{11}$ Arash Rezazadeh Kalebasty, ${ }^{12}$ Se Hoon Park, ${ }^{13}$ Boris Alekseev, ${ }^{14}$ Fabio Augusto Schutz, ${ }^{15}$ Jian-Ri Li, ${ }^{16}$ Almut Mecke, ${ }^{17}$ Sanjeev Mariathasan, ${ }^{18}$ AnnChristine Thåström, ${ }^{18}$ Enrique Grande ${ }^{19}$

${ }^{1}$ Hospital General Universitario Gregorio Marañón, Madrid, Spain; ${ }^{2}$ Icahn School of Medicine at Mount Sinai/Tisch Cancer Institute, New York, NY, USA; ${ }^{3}$ Charité University Hospital, Berlin, Germany and Department of Urology, Medical University, Vienna, Austria; ${ }^{4}$ Eastern Health/Monash University, Melbourne, VIC, Australia; ${ }^{5}$ National \& Kapodistrian University of Athens, Athens, Greece; ${ }^{6}$ Istituto Scientifico Romagnolo per lo Studio e la Cura dei Tumori (IRST), IRCCS, Meldola, Italy; ${ }^{7}$ Institute of Oncology, Ljubljana, Slovenia; ${ }^{8 K e i o}$ University, Tokyo, Japan; ${ }^{9}$ Catalan Institute of Oncology, IDIBELL, University of Barcelona, Barcelona, Spain; ${ }^{10}$ Istanbul Medeniyet University, Goztepe Research Hospital, Istanbul, Turkey; ${ }^{11}$ Istanbul University-Cerrahpasa, Cerrahpasa School of Medicine, Istanbul, Turkey; ${ }^{12}$ Norton Cancer Institute, Louisville, KY, USA; ${ }^{13}$ Sungkyunkwan University Samsung Medical Center, Seoul, Korea; ${ }^{14} \mathrm{P}$. Herzen Oncology Research Institute, Moscow, Russia; ${ }^{15}$ Beneficência Portuguesa de São Paulo, São Paulo, Brazil; ${ }^{16}$ Taichung Veterans General Hospital/HungKuang University, Taichung, Taiwan; ${ }^{17} \mathrm{~F}$. Hoffmann-La Roche Ltd, Basel, Switzerland; ${ }^{18}$ Genentech, Inc., South San Francisco, CA, USA; ${ }^{19} \mathrm{MD}$ Anderson Cancer Center Madrid, Madrid, Spain

\section{Background:}

- PD-L1 and PD-1 inhibitors are the first new systemic therapies for $\mathrm{mUC}$, both for $1 \mathrm{~L}$ treatment of cisplatin-ineligible patients and for patients experiencing disease progression despite platinum-based chemotherapy (cisplatin or carboplatin plus gemcitabine; plt/gem) $)^{1-8}$

- In July 2018, the FDA and EMA revised the $1 \mathrm{~L}$ label for atezolizumab (anti-PD-L1) and pembrolizumab (anti-PD-1) based on IDMC assessments ${ }^{9-12}$

- Here we report final PFS and interim OS results for IMvigor130, assessing atezolizumab in combination with $\mathrm{plt} / \mathrm{gem}$ vs placebo + plt/gem in $1 \mathrm{~L} \mathrm{mUC} \mathrm{(Fig.} \mathrm{1)}$

\section{Methods:}

- Eligibility criteria: locally advanced or metastatic UC, no prior systemic therapy in the metastatic setting, ECOG PS $\leq$ 2 , and $1 \mathrm{~L}$ platinum-eligible

- Stratification factors: PD-L1 IC status (IC0 vs IC1 vs IC2/3), Bajorin risk factor score including KPS $<80 \%$ vs $\geq 80 \%$ and presence of visceral metastases (0 vs 1 vs 2 and/or patients with liver metastases), investigator choice of plt/gem (cisplatin + gem or carboplatin + gem)

- Patients were randomised to Arm A (atezo + plt/gem), Arm B (atezo; added later per protocol amendment) or Arm C (placebo + plt/gem)

- Coprimary endpoints: investigator-assessed PFS (per RECIST 1.1) and OS (Arm A vs C, Arm B vs C)

Fig. 1

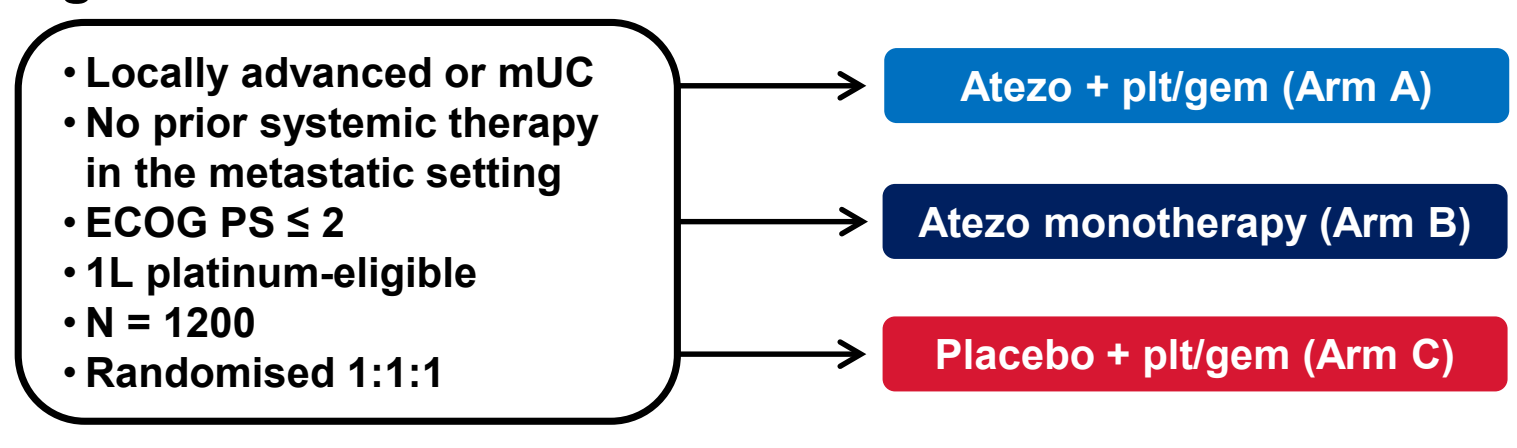

Results:

- 1213 patients were enrolled; 451 were randomly assigned to Arm A, 362 to Arm B, and 400 to Arm C (median survival follow-up for all patients was $11.8 \mathrm{mo}$ )

- Median final PFS was 8.2 mo for Arm A and 6.3 mo for Arm C (hazard ratio [HR], $0.82 ; 95 \% \mathrm{Cl}, 0.70$ to 0.96 ; 1-sided $P=0.007$ ) (Fig. 2)

- Median interim OS was 16.0 mo for Arm A and 13.4 mo for Arm $C$ but did not cross the prespecified efficacy boundary (HR, 0.83; 95\% Cl, 0.69 to 1.00, 1-sided $P=0.027$ ) (Fig. 3)

- The rate of investigator-assessed objective response was $47 \%$ (95\% Cl, 43\%-52\%) in Arm A, 23\% (19\%-28\%) in Arm B, and 44\% (39\%-49\%) in Arm C and complete response was seen in $13 \%$ of patients in Arm $A, 6 \%$ in Arm $B$, and $7 \%$ in Arm C (not shown)

- No new adverse event (AE) signals were observed, the combination safety profile was consistent with those of the individual therapeutic agents, and the safety profile was not markedly different from plt/gem alone (Table 1)

References: Grande et al. LBA14 ESMO 2019. 1. Gartrell et al. Urol Oncol 2017; 2. Balar et al. Lancet 2017; 3. Balar et al. Lancet Oncol 2017; 4. Powles et al. Lancet 2018; 5. Rosenberg et al. Lancet 2016; 6. Massard et al. J Clin Oncol 2016; 7. Sharma et al. Lancet Oncol 2017; 8. Apolo et al. J Clin Oncol 2017; 9. TECENTRIQ USPI 2019; 10. TECENTRIQ SmPC 2019; 11. KEYTRUDA USPI 2019; 12. KEYTRUDA SmPC 2019.
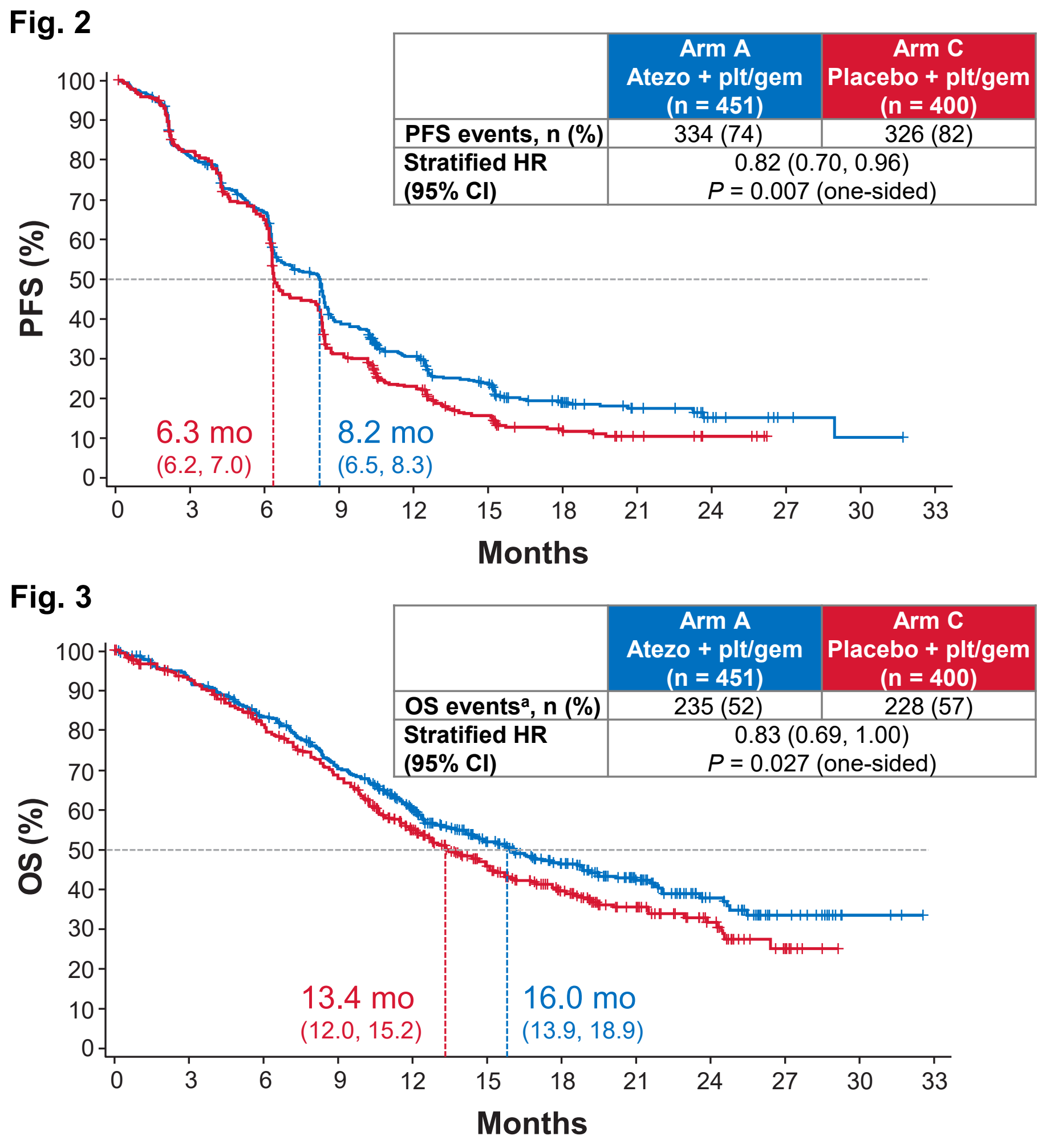

a $5 \%$ of patients from Arm A and $20 \%$ from Arm C received non-protocol immunotherapy.

Table 1

\begin{tabular}{|l|c|c|c|}
\hline AE, $\mathbf{n}(\%)$ & $\begin{array}{c}\text { Arm A } \\
\text { Atezo + plt/gem } \\
(\mathbf{n}=\mathbf{4 5 3 )}\end{array}$ & $\begin{array}{c}\text { Arm C } \\
\text { Placebo + plt/gem } \\
(\mathbf{n}=390)\end{array}$ & $\begin{array}{c}\text { Arm B } \\
\text { Atezo } \\
(\mathbf{n}=354)\end{array}$ \\
\hline Any grade, all cause & $451(100)$ & $386(99)$ & $329(93)$ \\
Grade 3-4 & $383(85)$ & $334(86)$ & $148(42)$ \\
Grade 5 & $29(6)$ & $20(5)$ & $28(8)$ \\
\hline Any grade, treatment related & $434(96)$ & $373(96)$ & $211(60)$ \\
Grade 3-4 & $367(81)$ & $315(81)$ & $54(15)$ \\
Grade 5 & $9(2)$ & $4(1)$ & $3(1)$ \\
\hline Any grade, serious & $234(52)$ & $191(49)$ & $152(43)$ \\
$\quad$ Treatment-related serious AEs & $144(32)$ & $101(26)$ & $44(12)$ \\
\hline Any grade leading to any treatment & $156(34)$ & $132(34)$ & $22(6)$ \\
discontinuation & $50(11)$ & $27(7)$ & $21(6)$ \\
Atezo or placebo & $53(12)$ & $52(13)$ & 0 \\
Cisplatin & $90(20)$ & $79(20)$ & $1(<1)$ \\
Carboplatin & $117(26)$ & $100(26)$ & $1(<1)$ \\
\hline Gemcitabine & $363(80)$ & $304(78)$ & $112(32)$ \\
\hline Any grade leading to any dose & & & \\
reduction or interruption & &
\end{tabular}

\section{Conclusions:}

- IMvigor130 is the first immune checkpoint inhibitor study to demonstrate an improvement in PFS over standard of care in $1 \mathrm{~L} \mathrm{mUC}$

- At this interim analysis, a numerically longer OS was observed with atezolizumab $+\mathrm{plt} / \mathrm{gem}$ vs placebo $+\mathrm{plt} / \mathrm{gem}$ but did not cross the prespecified interim efficacy boundary; follow-up will continue to final analysis

- Atezolizumab + plt/gem was well tolerated, with a safety profile consistent with each individual agent

- The results from IMvigor130 support atezolizumab + plt/gem as an important new treatment option for patients with untreated mUC 